Journal of

\section{Synchrotron Radiation}

ISSN 1600-5775

\section{current events}

This section carries events of interest to the synchrotron radiation community. Works for this section should be sent direct to the Current-Events Editors Friso van der Veen (friso.vanderveen@psi.ch) or Paul Zschack (pzschack@bnl.gov).

\section{Samuel Krinsky (1945-2014)}

Samuel Krinsky, recognized as a major driver for turning the National Synchrotron Light Source (NSLS) into a world-class user facility, passed away on 26 April 2014. He died at age 69. Krinsky was a senior physicist at Brookhaven National Laboratory.

Krinsky spent his entire career at Brookhaven, where he began learning accelerator physics under G. Kenneth Green and Renata Chasman. With John Blewett they formed the nucleus of the group that later became the National Synchrotron Light Source (NSLS) Department in 1982, the year NSLS began operations as a user facility. When Green and Chasman died in 1977, Krinsky was the only accelerator theorist on the team. One indication of his capability was his early design of a 'Super Photon' X-ray ring similar to what later on would be called a third-generation light source. This was deemed too risky at the time. Krinsky showed tremendous foresight; he was extremely interested in developing wigglers and undulators for use with electron accelerators as light sources, a passion that drove most of his career. He was a major driver for development of the global orbit feedback system, for which the group won an R\&D 100 award in 1989.

Krinsky also made outstanding contributions to both theory and experiments for an X-ray free-electron laser (XFEL), receiving the International FEL Prize in 2008. The culmination of his work on FEL gain was the paper co-authored by Li-Hua Yu, Krinsky and the late Robert Gluckstern (University of Maryland) on the determination of the gain in the exponential regime. This work had a significant impact on the advent of XFELs. Krinsky is also credited for conceiving of and building Brookhaven's Source Development Laboratory (SDL). The demonstration of outstanding qualities of a high-gain harmonic-

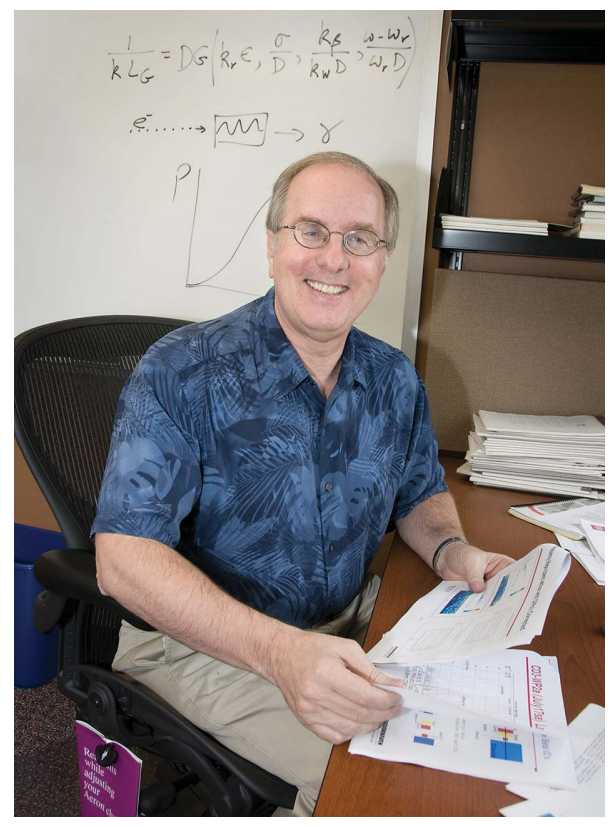

Samuel Krinsky. generation FEL operating in deep-ultraviolet mode at SDL has played an important role in seeding FEL projects worldwide.

At NSLS, Krinsky served in various leadership roles: head of the NSLS Accelerator R\&D from 1985 to 2001, deputy chair of the NSLS from 1986 to 2001, and manager of the FEL program from 1999 to 2002. In January 2008, he became group leader of Accelerator Physics in the NSLS-II Project to construct the National Synchrotron Light Source II. At the time of his death, Krinsky was managing the accelerator physics group within the Photon Sciences Directorate.

On a personal level, Samuel Krinsky is remembered for guiding his group and educating his scientists. His door was always open and his staff discussed their projects with him all day. There was no problem that Sam would not help by his advice, his wisdom and his experience. Family, friends and colleagues from across the US gathered at Brookhaven to pay tribute to Sam at a memorial symposium in October 2014. Krinsky is survived by his wife, Faith, and daughter, Sylvia.

\section{Leibniz Prize for DESY scientist Henry Chapman}

DESY scientist Henry Chapman will be awarded one of the prestigious Gottfried Wilhelm Leibniz Prizes 2015 by the German research foundation Deutsche Forschungsgemeinschaft (DFG). Henry Chapman receives the EUR 2.5 Million prize for his pioneering work in the development of femtosecond serial crystallography, enabling decoding of the structure of complex biomolecules in their natural environment with the help of an X-ray free-electron laser (XFEL).

Helmut Dosch, Chairman of the DESY Board of Directors, said "Chapman's pioneering work in this field will facilitate the analysis of molecular dynamics of highly complex systems. This will revolutionize biological structure research on a global scale and significantly influence its agenda in the coming decades."

Mainly with the use of synchrotron radiation, the structures of about 85000 proteins have been determined during the past decades. In all these cases it has been necessary to grow a single crystal of the protein of a size large enough (typically a few tens of micrometres) that diffraction patterns of sufficient quality are obtained. This

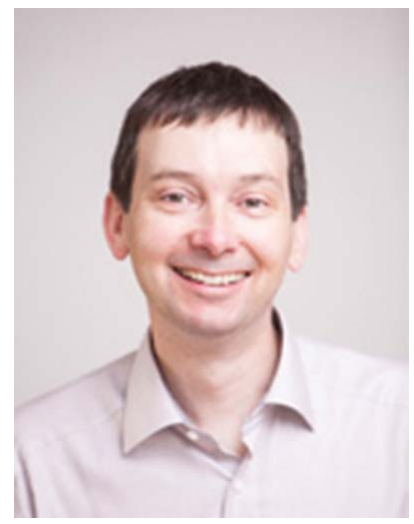

Henry Chapman. 


\section{current events}

process is often complex, sometimes impossible; moreover, the crystallization rips the biomolecule out of its natural environment. XFELs produce unprecedentedly brilliant and short X-ray flashes, opening up the possibility to measure diffraction patterns from tiny nanocrystals. Nanocrystals are easier to produce and generally have much fewer defects than larger ones. In serial femtosecond crystallography, a beam of these tiny crystals is traversed by X-ray laser light and hundreds of thousands of scattering images are taken, from which the protein structure can be reconstructed.

Chapman is honoured as a pioneer in the development of femtosecond serial crystallography. Using the method, he solved the structure of the Cathepsin B enzyme. Chapman's method of capturing the diffraction pattern before the nanocrystal is destroyed by the XFEL pulse can also create the right precondition for investigating samples almost in their natural environment. The latter may provide information about the structure and function of about 100000 biomolecules which have not yet been decoded.

\section{Innovation Award on Synchrotron Radiation for team at Swiss Light Source}

Each year, the Association of Friends of Helmholtz-Centre Berlin (HZB) bestows the Innovation Award on Synchrotron Radiation to a group or person from Europe that has made a significant contribution to the development of synchrotron radiation instrumentation or methodology. At the annual Users Meeting of HZB's large facilities, held in Berlin from 3 to 5 December 2014, the prize was awarded to Mirko Holler, Ana Diaz, Manuel Guizar-Sicairos and Jörg Raabe from the Paul Scherrer Institute. This group has set new standards in high-resolution three-dimensional hard X-ray microscopy by methodological developments in a coherent diffractive imaging approach called ptychography. The work was performed at the Swiss Light Source. The Innovation Award on Synchrotron Radiation is sponsored by SPECS GmbH and BESTEC GmbH.

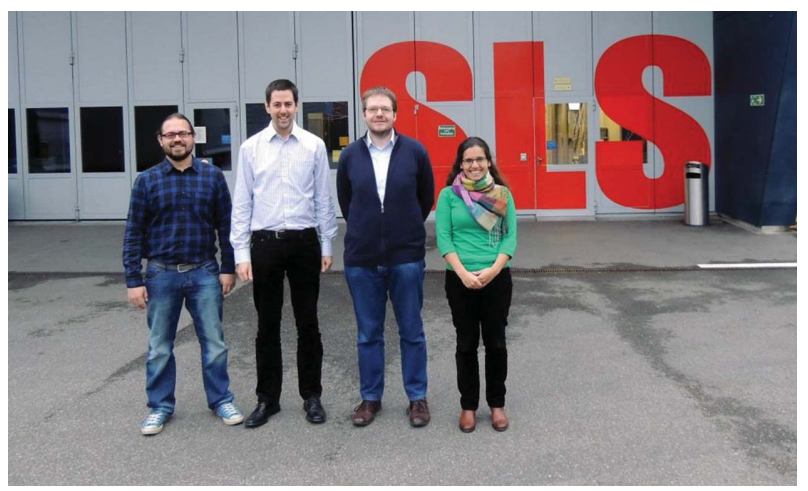

The prize-winning team in front of the Swiss Light Source. From left to right: Manuel Guizar-Sicairos, Mirko Holler, Jörg Raabe and Ana Diaz. 